\title{
Integral Equation Method for Scattering and Absorption of Electromagnetic Radiation by Thin Lossy Dielectric Discs
}

\author{
C. -M. Chu ANd Herschel WeIL \\ Department of Electrical and Computer Engineering \\ The University of Michigan, Ann Arbor, Michigan 48109
}

Received January 19, 1976

\begin{abstract}
An integral equation for the current induced in a dielectric body by incident electromagnetic radiation is solved for the case of a thin flat circular disc of homogeneous lossy dielectric material. The equation is reduced by using the method of moments to a linear algebraic matrix equation for the coefficients in an expansion of the current density as a linear combination of basis functions. The matrix elements consist of integrals involving the basis functions. The novel computational aspects of this work lie in the choice of basis functions and in the techniques which this choice enabled to handle singularities in the integrands when evaluating the matrix elements, to reduce the matrix size and to rigorously preserve symmetries in the matrix. Expressions are given for the scattering and absorption cross sections in terms of the expansion coefficients. The results are most suitable in the resonance range of wavelengths when the disc radius and free-space wavelength are of the same order of magnitude.
\end{abstract}

\section{INTRODUCTION}

Analytic solutions do not exist for the scattering and absorption of electromagnetic radiation by objects for which the vector wave equation is not separable. To handle other shapes it is necessary to use numerical techniques. One method which has been used with success for perfectly conducting bodies is to solve an integral equation for the surface currents induced by the incident wave; then to use these currents to compute the reradiated field [1-3]. There is no absorption in this idealized case.

In this paper we apply a similar technique to treat the scattering and absorption for thin dielectric discs. The induced currents are volume currents rather than surface currents, which greatly increases the complexity and size of the problem compared to the perfectly conducting case. We have used the method of moments to reduce the integral equation to a set of algebraic equations. This is similar to the methods used for perfect conductors in $[1,2]$, for example, but the functions we have used as basis functions in which to expand the unknown current density 
and as weighting functions when taking moments are more appropriate to the volume distribution problem than the functions used in the references. We have also used a different method than that in $[1,2]$ to approximate the integrals involved so as to preserve exactly the symmetry which should exist in the resulting matrix equations. Our choice of basis functions also aids in evaluation of the integrals in the vicinity of singularities which occur in the integrands and are chosen to be particularly appropriate for the thin disc problem.

The derivation of the integral equation and a number of computed scattering patterns plus the related total scattering and absorption data are given in a separate publication [4]. In this article we concentrate on the techniques used for solution of the integral equation. They form a variant of finite element methods as explained in Section 4.

Before proceeding we mention other work which has been published on the scattering by dielectric bodies. In [5] an integral equation for the induced field within the object is solved numerically by the point matching technique. In [6] an integral equation for induced "equivalent" surface-currents is solved by using orthogonal expansions of the fields. In [7] the vector wave differential equation is solved using an adaptation of Galerkin's method in which boundary conditions are enforced on the smallest spherical surface surrounding the object. In each of these papers the method was developed for arbitrary bodies and in none was it applied to thin discs.

\section{The Integral Equation and Reduction to Algebraic Equations}

The integral equation for induced current $\mathbf{J}$ in a homogeneous dielectric object of refractive index $n$ is

$$
\mathbf{E}^{i}=\frac{-1}{j \omega \epsilon_{0}}\left[\boldsymbol{L} \cdot \mathbf{J}-\frac{\mathbf{J}}{n^{2}-1}\right]
$$

where $\boldsymbol{L}$ is the self-adjoint integral operator defined by

$$
\begin{aligned}
L \cdot \mathbf{J}= & \iiint_{D} d v^{\prime} \mathbf{J}\left(\mathbf{R}^{\prime}\right) G\left(\mathbf{R}, \mathbf{R}^{\prime}\right) \\
& -\iint_{S} d s^{\prime} J\left(\mathbf{R}^{\prime}\right) \cdot \hat{n}^{\prime}\left(\mathbf{R}^{\prime}\right) \nabla G\left(\mathbf{R}, \mathbf{R}^{\prime}\right)
\end{aligned}
$$

$D$ and $S$ represent the volume and surface of the object and

$$
G=\exp \left(j\left|\mathbf{R}-\mathbf{R}^{\prime}\right|\right) /\left(4 \pi\left|\mathbf{R}-\mathbf{R}^{\prime}\right|\right) .
$$

All lengths are normalized by multiplication by $k=2 \pi / \lambda$ where $\lambda$ is the free space wavelength. The time dependence of the fields is assumed to be of the form $e^{j \omega t}$. 
The refractive index $n$ then has a negative imaginary part except for ideal nondissipative, nonconductive dielectrics.

To reduce (1) to a set of linear algebraic equations via the method of moments $\mathbf{J}(\mathbf{R})$ is expanded in a series of functions.

$$
\mathbf{J}(\mathbf{R})=-j \omega \epsilon_{0} \sum \alpha_{j} W_{j}(\mathbf{R}) .
$$

These expansion or basis functions should satisfy the requirement

$$
\begin{aligned}
\nabla \cdot \mathbf{W}_{t}=0 & \text { in } \mathrm{D} \\
\mathbf{W} \cdot \hat{n} \neq \mathbf{0} & \text { on } \mathrm{S}
\end{aligned}
$$

to ensure the similar properties for $\mathbf{J}(\mathbf{R})$ itself. Substitution of (4) into (1) yields

$$
\mathbf{E}^{i}=\sum_{i} \alpha_{i}\left[\boldsymbol{L} \cdot \mathbf{W}_{i}-\left(1 /\left(n^{2}-1\right)\right) \mathbf{W}_{i}\right] .
$$

Using the inner product

$$
\langle\mathbf{f}, \mathbf{g}\rangle=\iiint d v^{\prime} \mathbf{f}\left(\mathbf{R}^{\prime}\right) \cdot \mathbf{g}\left(\mathbf{R}^{\prime}\right)
$$

with (4) then yields an algebraic matrix equation to solve for the $\alpha_{i}$

$$
\left\langle\mathbf{W}_{j}, \mathbf{E}^{i}\right\rangle=-\sum_{i} \alpha_{i}\left[Z_{i j}-W_{i j} /\left(n^{2}-1\right)\right]
$$

where

$$
Z_{i j}=\left\langle\mathbf{W}_{i}, L \cdot \mathbf{W}_{j}\right\rangle, \quad \mathbf{W}_{i j}=\left\langle\mathbf{W}_{i}, \mathbf{W}_{j}\right\rangle .
$$

Equation (8) represents the moments of $\mathbf{E}^{i}$ with weight functions $\mathbf{W}_{j}$; i.e., the same functions as used for the basis functions.

The symmetric matrix $\left[Z_{i s}-W_{i j} /\left(n^{2}-1\right)\right]$ is termed an impedance matrix. All material effects are confined to the factor $\left(n^{2}-1\right)^{-1}$ while shape effects only are included in $Z_{i j}$ and $W_{i j}$. This separation proves of major practical importance because, to cut down on matrix size, our procedures lead to fairly complex and lengthy approximate expressions for the $Z_{i j}$; hence it is relatively expensive to load $Z_{i j}$ into the computer. Once this is accomplished, however, the stored $Z_{i j}$ and $W_{i j}$ may be used to investigate many materials.

We mention at this point that these approximate expressions for $Z_{i j}$ preserve the property $Z_{i j}=Z_{j i}$ exactly. By contrast the techniques used elsewhere as in $[1,2]$ do not preserve this symmetry. For problems treated in [2] numerical experimentation showed that the deviations from symmetry were small, but we have found that, for the dielectric disc problem the matrix evaluation methods of [2] lead to very pronounced assymetry. 
So far the analysis holds for any homogeneous dielectric body. The fact that our application is to thin discs is first used in choosing $\mathbf{W}_{i}(\mathbf{R})$ functions so as to minimize the required number of terms in the sum (4). This is explained in Section 4.

\section{Scattering and Absorption Cross-Sections}

Before going on to the problem of determining the $\alpha_{i}$ in the specific case of concern here, we give the formulas which express the scattering and absorption in terms of the expansion coefficients $\alpha_{j}$.

The scattering formulas are obtained by direct substitution of the current density into standard textbook formulas for the radiation field generated by a known current distribution. The bistatic cross section $\sigma_{B}(\theta, \phi)$ is defined in terms of a spherical coordinate system $(r, \theta, \phi)$ with origin in the object, for an incident plane-polarized plane wave as

$$
\sigma_{B}(\theta, \phi)=\frac{\text { radiated power/solid angle in direction }(\theta, \phi)}{\text { incident power/area }}
$$

for radiated power far from the scatterer. This becomes

$$
\sigma_{B}=(4 \pi k)^{-2}\left[\left|\sum_{i} \alpha_{i} \mathbf{P}_{i} \cdot \hat{\theta}\right|^{2}+\left|\sum_{i} \alpha_{i} \mathbf{P}_{i} \cdot \hat{\phi}\right|^{2}\right]
$$

where

$$
\mathbf{P}_{i}(\theta, \phi)=\iiint d v^{\prime} e^{j k f \cdot \mathbf{r}^{\prime}} \mathbf{W}_{i}\left(\mathbf{r}^{\prime}\right) .
$$

The absorption is found by integrating the ohmic loss in the body. The fact that the body is lossy is expressed by a finite conductivity $\sigma$ which renders the refractive index complex:

$$
n^{2}=\epsilon_{e}-j \sigma / \omega \epsilon_{0} \equiv n^{\prime}-j n^{\prime \prime} .
$$

The loss per unit volume is $\mathbf{J}_{c} \cdot \mathbf{J}_{\mathbf{c}}{ }^{*} /(2 \sigma)$ where $\mathbf{J}_{\mathbf{c}}$ is the part of the induced current which is conduction rather than polarization current, and the asterisk indicates complex conjugate. The integration of this expression over the volume yields the absorption cross-section.

$$
\begin{aligned}
\sigma_{a} & =\frac{\text { power dissipated in the material }}{\text { incident power/area }} \\
& =\frac{-2 n^{\prime} n^{\prime \prime}}{\left|n^{2}-1\right|^{2}} \frac{1}{k^{2}} \sum_{i} \sum_{j} \alpha_{i} \alpha_{j}{ }^{*} W_{i j} .
\end{aligned}
$$


Formulas for other measures of scattering and absorption, such as the matrix relating fields rather than powers and the remaining Stokes parameters could be derived in similar fashion.

The specific forms which (11) and (12) take for the $\mathbf{W}_{i}$ functions chosen in the sequel are listed in Appendix B.

\section{Choice of Basis Functions}

We use a cylindrical coordinate system $(\rho, \phi, z)$ with $z$ along the symmetry axis of the disc. The disc fills a region given by $-\delta \leqslant z \leqslant \delta, 0 \leqslant \rho \leqslant a$. Then, bearing (5) in mind we choose the $W_{i}$ from the following sets of functions. To most concisely express the induced current when $\mathbf{E}^{i}$ is perpendicular to $\hat{z}$ we use the TE mode

$$
\mathbf{W}_{E}^{(n, m)}=\nabla \times\left\{\hat{z} R(\rho) Z_{n}(z) \Phi_{m}(\phi)\right\}
$$

where

$$
Z_{n}(z)=z^{n} ; \quad \Phi_{m}(\Phi)=\sin m \phi \quad \text { or } \quad \Phi_{m}(\phi)=-\cos m \phi .
$$

The form of $Z_{n}(z)$ is chosen to permit easy integration over $z$ when evaluating the impedance expressions. In accord with the thin disc assumption only terms of order $\delta^{2}$ or greater will be kept in the impedance, hence only $n=0$ and 1 functions will be needed. The following odd TE modes resulting from the choice of $\sin m \phi$ in (13) result.

$$
\begin{aligned}
& \mathbf{W}_{E}^{(0, m)}=\hat{\rho}(m R(\rho) / \rho) \sin m \phi+\hat{\phi} R^{\prime}(\rho) \cos m \phi \\
& \mathbf{W}_{E}^{(1 . m)}=\hat{\rho}(m R(\rho) / \rho) z \sin m \phi+\hat{\phi} R^{\prime}(\rho) z \cos m \phi,
\end{aligned}
$$

etc.

When the incident wave is propagating either parallel or perpendicular to $\hat{z}$ and is plane polarized with $\mathbf{E}^{i}$ perpendicular to $\hat{z}$ only the one mode, $W_{E}^{(0,1)}$, is necessary.

Similarly, TM modes defined by

$$
\mathbf{W}_{M}^{(n, m)}=\nabla \times \mathbf{W}_{E}^{(n, m)}
$$

most concisely express the currents induced when $\mathbf{H}_{i} \perp \hat{z}$. The odd TM nodes are

$$
\begin{aligned}
\mathbf{W}_{M}^{(0, m)}= & \hat{z}\left[\left(R^{\prime}(\rho) / \rho\right)+R^{\prime \prime}(\rho)-\left(m^{2} R(\rho) / \rho^{2}\right)\right] \sin m \phi \\
\mathbf{W}_{M}^{(1, m)}= & \hat{\rho} R^{\prime}(\rho) \sin m \phi+\hat{\phi}(m R(\rho) / \rho) \cos m \phi \\
& +\hat{z}\left[-\left(R^{\prime}(\rho) / \rho\right)-R^{\prime}(\rho)+\left(m^{2} R(\rho) / \rho^{2}\right)\right] \sin m \phi .
\end{aligned}
$$

etc. 
For even modes $m \phi$ is to be replaced by $m \phi+(\pi / 2)$ in (14) and (17). When neither $\mathbf{E}^{i}$ nor $\mathbf{H}^{i}$ are perpendicular to $\hat{z}$, a combination of TE and TM mode $\mathbf{W}$ functions will be required.

With these $\mathbf{W}$ functions there is no coupling between the different values of $m$; that is, the impedance terms corresponding to use of $\mathbf{W}^{(n, m)}$ and $\mathbf{W}^{\left(n, m^{\prime}\right)}$ are zero when $m \neq m^{\prime}$.

We will choose a set of $R(\rho)$ functions $\left\{\boldsymbol{R}_{i}(\rho)\right\}$ which will generate corresponding sets of basis functions $\left\{\mathbf{W}_{E i}^{(n, m)}\right\}$ and $\left\{\mathbf{W}_{M i}^{(n, m)}\right\}$. Each $R_{i}(\rho)$ is chosen to be zero over all but a fractional part of the interval $0 \leqslant \rho \leqslant a$. The subintervals are taken small enough to permit the integrals to be evaluated by approximate means with adequate accuracy. A trade-off is involved in the choice of interval size; larger intervals require a lesser number of basis functions hence smaller impedance matrices, but on the other hand the larger intervals lead to longer, more complex expressions to express the individual matrix elements with sufficient accuracy. We avoid an excessively large number of intervals with the choice of $R_{i}(\rho)$ given below. We are then able to treat the singularity region to desired accuracy by reducing the problem to the singularity integration for a statics problem plus correction terms, all of which are evaluated analytically. We do end up with lengthy expressions for the matrix elements as we mentioned earlier.

In the choice of $R_{i}(\rho)$ we deviate from past practice in the literature [1,2] where perfectly conducting objects were considered and linear triangular functions were used. However, one needs second derivatives of $R(\rho)$ for the dielectric case. For the triangle functions these would be impulse functions which would correspond to simple pointwise sampling of these terms. Therefore, for greater accuracy we choose instead symmetric functions made of segments of second order polynomials. Specifically, we divide the interval $0 \leqslant \rho \leqslant a$ into $N$ contiguous segments of length $a / N$. Then the functions $R_{i}(\rho)$ are the spline functions illustrated schematically in Fig. 1 and defined for $i=1,2, \ldots, N$ by (18).

$$
\begin{array}{rc}
(a / N)(i-1)<\rho<(a / N) i, & (1 / 2)(N / a)^{2}[\rho-(a / N)(i-1)]^{2} \\
(a / N) i \leqslant \rho \leqslant(a / N)(i+1), & -1+2(N / a)[\rho-(a / N)(i-1)] \\
& -(1 / 2)(N / a)^{2}[\rho-(i-1)(a / N)]^{2} \\
(a / N)(i+1) \leqslant \rho<(a / N)(i+2), & -1+2(a / N)[(a / N)(i+3)-\rho] \\
& -(1 / 2)(N / a)^{2}[(a / N)(i+3)-\rho]^{2} \\
(a / N)(i+2) \leqslant \rho \leqslant(a / N)(i+3), & (1 / 2)(N / a)^{2}[(a / N)(i+3)-\rho]^{2}
\end{array}
$$

provided $\rho \leqslant a$. For $\rho>a, R_{i}(\rho)=0$. This treatment of the radially dependent part of the problem is typical of techniques used in finite element methods. Note that there is an overlapping of the $R_{i}$ 's in the integrations for $Z_{i j}$ and $W_{i j}$. 


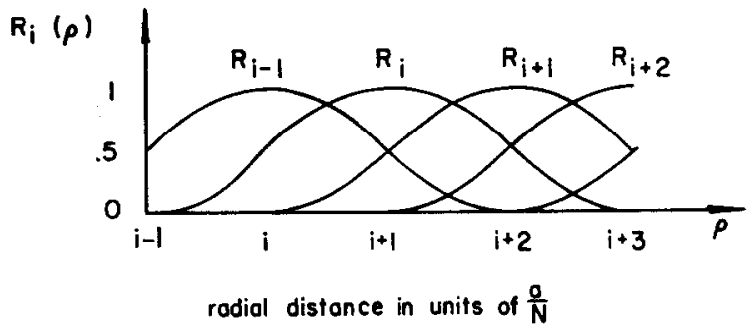

FIG. 1. Illustration of the function defined in Eq. (18).

Numerical evaluation of the integrals is facilitated by use of average values over short intervals for $R_{i}(\rho)$, and functions involving $R_{i}(\rho)$, and its derivatives. For this each interval $(a / N)(i-1) \leqslant \rho \leqslant(a / N)(i+3)$ is subdivided into eight equal parts denoted by $p=1, \ldots, 8$ each extending from

$$
\alpha_{i p}=(a / N)(i-1+((p-1) / 2))
$$

to

$$
\beta_{i p}=(a / N)(i-1+(p / 2))
$$

TABLE I

Mean Values of the Radial Functions

\begin{tabular}{lrrrrc}
$a$ & $A_{i a}$ & $B_{i q}$ & $C_{i q}$ & $D_{i q}$ & $E_{i q}$ \\
\hline 1 & $\frac{1}{24}$ & $-\frac{1}{6}+\frac{1}{4} i$ & $\frac{1}{4}\left(\frac{N}{a}\right)$ & $\frac{N}{a}\left(i-\frac{3}{4}\right)$ & $\frac{N}{a}\left[\frac{5}{8}-\frac{i}{2}+(i-1)^{2} \operatorname{Ln} \frac{2 i-1}{2 i-2}\right]$ \\
2 & $\frac{7}{24}$ & $-\frac{1}{6}+\frac{3}{4} i$ & $\frac{3}{4}\left(\frac{N}{a}\right)$ & $\frac{N}{a}\left(i-\frac{1}{4}\right)$ & $\frac{N}{a}\left[\frac{7}{8}-\frac{i}{2}+(i-1)^{2} \operatorname{Ln} \frac{2 i}{2 i-1}\right]$ \\
3 & $\frac{17}{24}$ & $+\frac{1}{6}+\frac{3}{4} i$ & $\frac{3}{4}\left(\frac{N}{a}\right)$ & $-\frac{N}{a}\left(i+\frac{1}{4}\right)$ & $\frac{N}{a}\left[\frac{7}{8}+\frac{i}{2}-\left(i^{2}+2 i-1\right) \operatorname{Ln} \frac{2 i+1}{2 i}\right]$ \\
4 & $\frac{23}{24}$ & $+\frac{1}{6}+\frac{1}{4} i$ & $\frac{1}{4}\left(\frac{N}{a}\right)$ & $-\frac{N}{a}\left(i+\frac{3}{4}\right)$ & $\frac{N}{a}\left[\frac{5}{8}+\frac{i}{2}-\left(i^{2}+2 i-1\right) \operatorname{Ln} \frac{2 i+2}{2 i+1}\right]$ \\
5 & $\frac{23}{24}$ & $-\frac{1}{3}-\frac{1}{4} i$ & $-\frac{1}{4}\left(\frac{N}{a}\right)$ & $-\frac{N}{a}\left(i+\frac{5}{4}\right)$ & $\frac{N}{a}\left[\frac{3}{8}+\frac{i}{2}-\left(i^{2}+2 i-1\right) \operatorname{Ln} \frac{2 i+3}{2 i+2}\right]$ \\
6 & $\frac{17}{24}$ & $-\frac{4}{3}-\frac{3}{4} i$ & $-\frac{3}{4}\left(\frac{N}{a}\right)$ & $-\frac{N}{a}\left(i+\frac{7}{4}\right)$ & $\frac{N}{a}\left[\frac{5}{8}+\frac{i}{2}-\left(i^{2}+2 i-1\right) \operatorname{Ln} \frac{2 i+3}{2 i+2}\right]$ \\
7 & $\frac{7}{24}$ & $-\frac{5}{3}-\frac{3}{4} i$ & $-\frac{3}{4}\left(\frac{N}{a}\right)$ & $\frac{N}{a}\left(i+\frac{9}{4}\right)$ & $\frac{N}{a}\left[-\frac{15}{8}-\frac{i}{2}+(i+3)^{2} \subseteq \operatorname{Cn} \frac{2 i+5}{2 i+4}\right]$ \\
8 & $\frac{1}{24}$ & $-\frac{2}{3}-\frac{1}{4} i$ & $-\frac{1}{4}\left(\frac{N}{a}\right)$ & $\frac{N}{a}\left(i+\frac{11}{4}\right)$ & $\frac{N}{a}\left[-\frac{13}{8}-\frac{i}{2}+(i+3)^{2} \operatorname{Ln} \frac{2 i+6}{2 i+5}\right]$
\end{tabular}


and centered on $\gamma_{i p}$. We define averages over these intervals

$$
A_{i n} \equiv\left\langle R_{i}(\rho)\right\rangle_{p} \equiv(a / 2 N)^{-1} \int_{\alpha_{i p}}^{\beta_{i p}} R_{i}(\rho) d \rho
$$

and similarly

$$
\begin{aligned}
B_{i p} & \equiv\left\langle R_{i}{ }^{\prime}(\rho)\right\rangle_{p}, \quad C_{i p} \equiv\left\langle R_{i}(\rho)\right\rangle_{p}, \\
D_{i p} & \equiv\left\langle R_{i}^{\prime \prime}(\rho)\right\rangle_{p}, \quad E_{i p} \equiv\left\langle R_{i}(\rho) / \rho\right\rangle_{p} .
\end{aligned}
$$

Table I lists the explicit formulas for $A_{i p} \cdots E_{i p}$.

\section{Evaluation of Impedance for Thin Flat Circular Discs}

The $W_{i j}$ each involve only one volume integral and can be evaluated analytically with our choice of polynomial functions for the $R_{i}(\rho)$ by straightforward integration. The results are given in Appendix A.

The $Z_{i j}$ each involve double volume or surface integrals. They are carried out by partly numerical and partly analytical methods.

To begin with the double integration over $\rho$ and $\phi$ is reduced, using trigonometric identities, to integrals of the types

$$
\int_{0}^{2 \pi} d \phi \int_{0}^{2 \pi} d u\left(\begin{array}{c}
\cos m u \cos u \\
\sin m u \sin u
\end{array}\right) G(u)
$$

where $G(u)$ represents the dependance of the Green's function on $u \equiv \phi-\phi^{\prime}$. $G(u)$ is an even function of $u$. The $u$ integration is carried out numerically as a sum over $L$ equal angular sectors using

$$
\int_{0}^{2 \pi} g(u) d u=(L / 2 \pi) \sum_{i=1}^{L} g\left(u_{t}\right)
$$

where the $u_{l}$ are midpoints of the sectors. For greater accuracy, the $l=1$ interval where $G(u)$ is rapidly varying (near the integrable singularity for $u=0$ ) may be further subdivided.

For the $\rho$ integrations we split the intervals in which $\boldsymbol{R}_{i}(\rho)$ and $\boldsymbol{R}_{j}(\rho)$ overlap into the subintervals centered on $\rho=\gamma_{i p}$ which are described by (19). Then in each subinterval we replace the $R_{i}(\rho), R_{i}^{\prime}(\rho)$ etc. by the averages listed in Table I. With these approximations the $Z_{i j}$ integration reduces to linear combinations of terms of the form

$$
I\left(\rho, \rho^{\prime}, u_{l}\right)=\int_{-\delta / 2}^{\delta / 2} d z^{\prime} \int_{-\delta / 2}^{\delta / 2} d z G\left(z, z^{\prime}, \rho, \rho^{\prime}, u_{l}\right)
$$


The function $G$ is given by (3) and may be rewritten as

$$
G=\frac{1}{4 \pi} \frac{\exp \left(-j\left(D^{2}+Z^{2}\right)^{1 / 2}\right)}{\left(D^{2}+Z^{2}\right)^{1 / 2}}
$$

where the transverse distance $D$ is the projection of $\left|R-R^{\prime}\right|$ on a plane perpendicular to the $z$-axis;

$$
D=D\left(\rho, \rho^{\prime}, u_{l}\right)=\left(\rho^{2}+\rho^{1 / 2}-2 \rho \rho^{\prime} \cos u_{l}\right)^{1 / 2} .
$$

For a specific example consider the impedance $Z_{i j}^{11}$ due to the zeroth order TE modes $W_{E i}^{(0, M)}$ and $W_{E j}^{(0, M)}$. With the steps and notation so far introduced we have

$$
\begin{aligned}
Z_{i j}^{11} \sim & \left(2 \pi^{2} / L\right) \sum_{l=1}^{L} \sum_{p=1}^{p_{0}} \sum_{q=1}^{q_{\nu}} \int_{\alpha_{i p}}^{\beta_{i p}} d \rho \int_{\alpha_{j q}}^{\beta_{j q}} d \rho^{\prime}\left\{\cos m u_{l} \cos u_{l}\left(m^{2} A_{i p} A_{j q}+B_{i p} B_{j q}\right)\right. \\
& \left.+m \sin m u_{l} \sin u_{l}\left(A_{i p} B_{j q}+B_{i p} A_{j q}\right)\right\} I\left(\rho, \rho^{\prime}, u_{l}\right) \\
& -\left(2 \pi^{2} / L\right) m^{2}\left((1 / 2) \delta_{N}^{i}+\delta_{N-1}^{i}+(1 / 2) \delta_{N-2}^{i}\right)\left((1 / 2) \delta_{N}^{j}+\delta_{N-1}^{j}-(1 / 2) \delta_{N-2}^{j}\right) \\
& \times \cos m u_{l} I\left(a, a, u_{l}\right) .
\end{aligned}
$$

Here $\delta_{\alpha}{ }^{\beta}=1$ if $\alpha=\beta,=0$ if $\alpha \neq \beta$. The values of $p_{0}$ and $q_{0}$ are given by Table II. They are determined by the number of overlapping subintervals (19) which lie within the integration range $0<\rho<a$. To evaluate the integrals (24) we introduce

$$
\bar{D}=D\left(\gamma_{i p}, \gamma_{\alpha q}, u_{l}\right)
$$

$\bar{D}$ is the transverse distance between center points of subintervals defined by (19). Then noting that $Z=\left|z-z^{\prime}\right|<\delta \ll 1$ and $|D-\bar{D}| \ll 1$ we approximate the function $G$ of (26) as follows.

$$
\begin{aligned}
G & =(1 / 4 \pi) \exp (-j \bar{D}) \frac{\exp \left(-j\left(D^{2}+Z^{2}\right)^{1 / 2}-j \bar{D}\right)}{\left(D^{2}+Z^{2}\right)^{1 / 2}} \\
& \sim \frac{\exp (-j \bar{D})}{4 \pi}\left(\frac{1+j \bar{D}}{\left(D^{2}+Z^{2}\right)^{1 / 2}}-j\right) .
\end{aligned}
$$

TABLE II

Summation Limits $p_{0}$ and $q_{0}$

\begin{tabular}{cc}
\hline$i$ & $p_{0}, q_{0}$ \\
\hline$\leqslant N-3$ & 8 \\
$=N-2$ & 6 \\
$=N-1$ & 4 \\
$=N$ & 2 \\
\hline
\end{tabular}


With this approximation (25) may be evaluated correct to terms of order $\delta$ by straightforward integration. By use of (29) we have reduced the integrations to that of the statics problem where the Green's function $G$ is simply $\left(D^{2}+Z^{2}\right)^{-1 / 2}$.

The final forms we obtained for $Z_{i j}^{11}$ are given in Appendix A.

\section{Numerical Evaluation}

For a given incident wave direction and polarization the left side of $(8),\left\langle\mathbf{W}_{i}, \mathbf{E}^{i}\right\rangle$, is readily evaluated analytically with the $\mathbf{W}_{i}$ function we have introduced. We have done this and worked out numerical results for broadside and edge on incidence when $\mathbf{E}^{i}$ is parallel to the plane of the disc and $a \leqslant 6$. The formulas for $\left\langle\mathbf{W}_{j}, \mathbf{E}^{i}\right\rangle$ are given in Appendix $A$ and numerical results are given in [4]. As pointed out in Section 4 only one set of basis functions is needed for these cases, the lowest order TE modes corresponding to $W_{E}^{(0)}$ evaluated for the members of the set $\left\{R_{i}\right\}$. A study of these cases thus can be looked at as a study of the building blocks or submatrices from which to handle more general cases. We used matrix inversion by converting to upper and lower triangular matrices and experimented with the effects of angular and radial interval sizes and the use of double precision airthmetic.

We found very little change in the scattering results when we used more than 10 radial and 12 angular intervals in the range $0^{\circ}<u<90^{\circ}$ (all that is needed because of symmetries) using single precision arithmetic. For the case of incidence edge-on, letting $m$ be the nearest integer exceeding $k a+1$ was sufficient. For this small number of radial intervals the matrix inversion costs were trivial, almost all the computation costs went into loading matrix $Z_{i j}$ which, as we pointed out in Section 3 is independent of the disc material and so may be stored and used for many materials. This fact and the very small matrix size which the experiment indicated is needed for each submatrix corresponding to an $m$ value indicates that the computing costs will not be excessive for more complicated cases involving irradiating fields incident in arbitrary directions or involving more than one disc.

We should point out that our method was designed for use with thin lossy dielectric discs, not highly conducting ones. The induced volume distribution of current is assumed to be uniform across the thin dimension $\delta$ of the disc; consequently it, the resultant fields and the cross sections all vanish as $\delta \rightarrow 0$. On the other hand, for highly conducting discs $n \sim n_{0}(1-j), \lim _{\sigma \rightarrow \infty} n_{0}=\infty$ and the induced current is confined to the surface. In fact, for $\sigma=\infty$, since the induced current is a purc surface current, even a disc of zero thickness yiclds a nonzero scattering cross section - which in fact will approximate well the results for highly conducting thin discs. On the other hand, for $n_{0} \gtrsim 10^{6}$ brief numerical experimentation using our procedures showed the broadside back-scatter to be roughly propor- 
tional to $\delta^{2}$. For a thickness of $\delta=0.1$ and $n_{0} \geqslant 10^{6}$ broadside backscatter computed by our methods $k a \leqslant 6$ increases radically relative to the values for low refractive index; the backscattering cross section versus $k a$ curve follows the result computed in [8] for infinitely thin perfectly conducting discs but is roughly a factor of four smaller.

\section{APPENDIX A: IMPEDANCE AND EXCITATION FORMULAS FOR LOWEST ORDER TE MODES}

Impedances

$$
\begin{aligned}
Z_{i j}^{11(m)} \simeq & \left(\pi \delta^{2} / 2 L\right) \sum_{l=1}^{L} \sum_{p=1}^{p_{\nu}} \sum_{q=1}^{q_{\nu}} e^{-j \bar{D}}\left\{(1+j \bar{D}) \mathscr{F}_{l}-j(a / 2 N)^{2}\right\} \\
& \times\left\{\cos m u_{l} \cos u_{l}\left(m^{2} A_{i p} A_{j q}+B_{i p} B_{j q}\right)\right. \\
& \left.+\sin m u_{l} \sin u_{l}\left(m A_{i p} B_{j q}+m B_{i p} A_{j q}\right)\right\} \\
& -m^{2} \cos m u_{l}\left((1 / 2) \delta_{N}^{i}+\delta_{N-1}^{i}+(1 / 2) \delta_{N-2}^{j}\right) \\
& \cdot\left((1 / 2) \delta_{N}^{j}+\delta_{N-1}^{j}+(1 / 2) \delta_{N-2}^{j}\right) e^{-j\left(2 a^{2}\left(1-\cos u_{l}\right)\right)^{1 / 2}} K\left(u_{l}\right)
\end{aligned}
$$

where

$$
\begin{aligned}
\mathscr{F}_{l}(i, j, p, q) & =F_{l}\left(\beta_{i p}, \beta_{j q}\right)+F_{l}\left(\alpha_{i p}, \alpha_{j q}\right)-F_{l}\left(\beta_{i p}, \alpha_{j q}\right)-F_{l}\left(\alpha_{i p}, \beta_{j q}\right), \\
F_{l}\left(r, r^{\prime}\right)= & r^{\prime} \ln \left(r-r^{\prime} \cos u_{l}+R\right)+r \ln \left(r^{\prime}-r \cos u_{l}+R\right) \\
& +2\left(\sin u_{l} / \delta\right)\left[r^{\prime 2} \tan ^{-1}\left(r^{\prime} \delta \sin u_{l} /\left(\left(r-r^{\prime} \cos u_{l}\right) R+d^{2}\right)\right)\right. \\
& \left.+r^{2} \tan ^{-1}\left(r \delta \sin u_{l} /\left(\left(r^{\prime}-r \cos u_{l}\right) R+d^{2}\right)\right)\right] \\
& -\left(\sin ^{2} u_{l} / \delta^{2}\right)\left[r^{\prime 3} \ln \left(\left(r-r^{\prime} \cos u_{l}+R\right) /\left(r-r^{\prime} \cos u_{l}+d\right)\right)\right. \\
& \left.+r^{3} \ln \left(\left(r^{\prime}-r \cos u_{l}+R\right) /\left(r^{\prime}-r \cos u_{l}+d\right)\right)\right] \\
& +\left(d^{2} \cos u_{l}-2 r r^{\prime} \sin u_{l}\right)(R-d-2 \delta \ln ((\delta+R) / d)) / \delta^{2}
\end{aligned}
$$

and

$$
\begin{aligned}
K\left(u_{l}\right)= & (2 / \delta) \ln \left(\left(2 a^{2}\left(1-\cos u_{l}\right)+\delta^{2}\right)^{1 / 2} /\left(2 a^{2}\left(1-\cos u_{l}\right)\right)^{1 / 2}\right) \\
& -\left(2 / \delta^{2}\right)\left[\left(2 a^{2}\left(1-\cos u_{l}\right)+\delta^{2}\right)^{1 / 2}-\left(2 a^{2}\left(1-\cos u_{l}\right)\right)^{1 / 2}\right]-j
\end{aligned}
$$

The values of $p_{0}$ and $q_{0}$ are given in Table II.

Formulas for $W_{i j}^{m}=W_{j i}^{m} \equiv\left\langle W_{E i}^{(0, m)}, W_{E j}^{(0, m)}\right\rangle$ are as follows. $W_{i j}^{m}$ is nonvanishing if $|i-j|<3$. We have

$$
\begin{array}{rlrl}
W_{i, *+1}^{m}=W_{i+1, i}^{m}, \quad W_{i, i+2}^{m}=W_{i+2, i}^{m}, & & W_{i+3, i}^{m}=W_{i, i+3}^{m}, \\
W_{i, i}^{m} & =f_{1}^{m}(i) & & i=N \\
& =f_{1}^{m}+f_{2}^{m} & & i=N-1 \\
& =f_{1}^{m}+f_{2}^{m}+f_{3}{ }^{m} & & i=N-2 \\
& =f_{1}{ }^{m}+f_{2}{ }^{m}+f_{3}{ }^{m}+f_{4}{ }^{m} & & i \leqslant N-3,
\end{array}
$$




$$
\begin{aligned}
f_{1}^{m}(i)= & \pi \delta\left\{( m ^ { 2 } / 4 ) \left[(1 / 4)-((i-1) / 3)+\left((i-1)^{2} / 2\right)-(i-1)^{3}\right.\right. \\
& \left.\left.+(i-1)^{4} \operatorname{Ln}(i /(i-1))\right]+(1 / 4)+((i-1) / 3)\right\} \\
f_{2}^{m}(i)= & \pi \delta\left\{( m ^ { 2 } / 4 ) \left[(7 / 4)+(11 / 3)(i+1)-\left((i+1)^{2} / 2\right)-(i+1)^{3}\right.\right. \\
& \left.\left.-\left[(i+1)^{4}-4(i+1)^{2}+4\right] \operatorname{Ln}(i /(i+1))\right]+((i+1) / 3)-(1 / 4)\right\}, \quad(\mathrm{A} 6) \\
f_{3}{ }^{m}(i)= & \pi \delta\left\{( m ^ { 2 } / 4 ) \left[-(7 / 4)+11((i+1) / 3)+\left((i+1)^{2} / 2\right)-(i+1)^{3}\right.\right. \\
& \left.\left.\left.-\left[(i+1)^{4}-4(i+1)^{2}+4\right)\right] \operatorname{Ln}((i+2) /(i+1))\right]+(1 / 4)+((i+1) / 3)\right\} \\
f_{4}^{m}(i)=\pi & \pi \delta\left\{( m ^ { 2 } / 4 ) \left[-(1 / 4)-(1 / 3)(i+3)-(1 / 2)(i+3)^{2}-(i+3)^{3}\right.\right. \\
& \left.\left.-(i+3)^{4} \operatorname{Ln}((i+2) /(i+3))\right]+((i+3) / 3)-(1 / 4)\right\} \\
W_{i, i+1}^{m}= & g_{1}{ }^{m}(i) \\
= & g_{1}{ }^{m}(i)+g_{2}{ }^{m}(i) \quad i=N-1 \\
= & g_{1}{ }^{m}(i)+g_{2}{ }^{m}(i)+g_{3}{ }^{m}(i) \quad i \leqslant N-3
\end{aligned}
$$$$
g_{1}^{m}(i)=\pi \delta\left\{\left(m^{2} / 4\right)\left[(11 / 12)-(5 / 3) i+(3 / 2) i^{2}+i^{3}-\left(i^{2}-2 i^{3}-i^{4}\right) \operatorname{Ln}((1+i) / i)\right]\right.
$$$$
+(1 / 12)+(i / 6)\}
$$$$
g_{\mathrm{a}}{ }^{m}(i)=\pi \delta\left\{( m ^ { 2 } / 4 ) \left[(25 / 12)+(11 / 3)(i+1)-(3 / 2)(i+1)^{2}-(i+1)^{3}\right.\right.
$$$$
\left.+\left[2-4(i+1)-3(i+1)^{2}+2(i+1)^{3}+(i+1)^{4}\right] \operatorname{Ln}((i+2) /(i+1))\right]
$$$$
-(1 / 12)-((i+1) / 6)\}
$$$$
g_{3}{ }^{m}(i)=\pi \delta\left\{( m ^ { 2 } / 4 ) \left[-(37 / 12)-(5 / 3)(i+2)+(3 / 2)(i+2)^{2}+(i+2)^{3}\right.\right.
$$$$
\left.+\left[2+4(i+2)+(i+2)^{2}-2(i+2)^{3}-(i+2)^{4}\right] \operatorname{Ln}((i+3) /(i+2))\right]
$$$$
+(1 / 12)+((i+2) / 6)
$$$$
\begin{aligned}
W_{i, i+2}^{m} & =h_{1}(i), & & i=N-2 \\
& =h_{1}(i)+h_{2}(i), & & i \leqslant N-3,
\end{aligned}
$$$$
h_{1}{ }^{m}(i)=\pi \delta\left\{( m ^ { 2 } / 4 ) \left[(3 / 4)-(5 / 3)(i+1)-\left((i+1)^{2} / 2\right)+(i+1)^{3}\right.\right.
$$$$
\left.\left.+\left[2(i+1)^{2}-(i+1)^{4}\right] \operatorname{Ln}((i+2) /(i+1))\right]-(1 / 4)-((i+1) / 3)\right\},
$$$$
h_{2}{ }^{m}(i)=\pi \delta\left\{( m ^ { 2 } / 4 ) \left[-(3 / 4)-(5 / 3)(i+3)+\left((i+3)^{2} / 2\right)+(i+3)^{3}\right.\right.
$$$$
\left.\left.+\left[(i+3)^{4}-2(i+3)^{2}\right] \operatorname{Ln}((i+2) /(i+3))\right]-((i+3) / 3)+(1 / 4)\right\} \text {, }
$$

$$
\begin{aligned}
W_{i, i+3}^{m}= & \pi \delta\left\{( m ^ { 2 } / 4 ) \left[(1 / 12)-(1 / 3)(i+2)-(3 / 2)(i+2)^{2}-(i+2)^{3}\right.\right. \\
& \left.+\left[(i+2)^{4}+2(i+2)^{3}+(i+2)^{2}\right] \operatorname{Ln}((i+3) /(i+2))\right] \\
& -(5 / 12)-(i / 6)\}, \quad i \leqslant N-3
\end{aligned}
$$




\section{Excitations}

We give the formulas for $I_{i}^{(m)} \equiv\left\langle W_{E i}^{(0, m)}, \mathbf{E}^{i}\right\rangle$ for two cases.

(a) Broadside incidence $E^{\imath}=\hat{y} e^{-j z}$.

The odd TE mode with $m=1$ is the only one excited. To $O\left(\delta^{2}\right)$

$$
\begin{aligned}
I_{N}^{(1)} & =\delta \pi a / 2 \\
I_{N-1}^{(1)} & =\delta \pi a \\
I_{N-2}^{(1)} & =\delta \pi a / 2 \\
I_{i}^{(1)} & =0, \quad i<N-2 \\
I_{i}^{(m)} & =0, \quad m>1
\end{aligned}
$$

(b) Edge-on incidence, $\mathbf{E}$ parallel to the disc surface, $\mathbf{E}^{i}=\hat{y} e^{-j x}$.

The odd TE modes for all $m$ are excited

$$
I_{i}^{(m)}=\pi \delta(-j)^{m-1}(a / 2 N) \sum_{p=1}^{p_{0}}\left(m A_{i p}+B_{i p}\right) J_{m-1}\left(\gamma_{i p}\right)+\left(m A_{i p}-B_{i p}\right) J_{m+1}\left(\gamma_{i p}\right)
$$

Here the $J_{n}\left(\gamma_{i p}\right)$ are Bessel functions.

\section{APPENDIX B: CROSS SECTION Formulas}

For $W_{E}^{(0, m)}$ the bistatic cross section (11) reduces to

$$
\sigma_{B}(\theta, \phi)=(a \delta / 8 N k)^{2} \sum_{m}\left[\left|\cos ^{2} \theta \sin ^{2} m \phi\left(f_{m}-g_{m}\right)\right|^{2}+\left|\cos ^{2} m \phi\left(f_{m}+g_{m}\right)\right|^{2}\right]
$$

where

$$
\begin{aligned}
& f_{m}=\sum_{i=1}^{N} \sum_{q=1}^{q_{0}} \alpha_{i}\left(m A_{i q}+B_{i q}\right) J_{m-1}\left(\gamma_{i q} \sin \theta\right) \\
& g_{m}=\sum_{i=1} \sum_{q=1} \alpha_{i}\left(m A_{i q}-B_{i q}\right) J_{m+1}\left(\gamma_{i q} \sin \theta\right) .
\end{aligned}
$$

Evaluating (12) gives the absorption cross section

$$
\begin{aligned}
\sigma_{a}= & \left(-2 n^{\prime} n^{\prime \prime} /\left|\left(n^{2}-1\right)\right|^{2} k^{2}\right) \sum_{m}\left\{\sum_{i=1}^{N} \alpha_{i} \alpha_{i}^{*} W_{i, i}^{m}+\sum_{i=1}^{N-1}\left(\alpha_{i} \alpha_{i+1}+\alpha_{i}^{*} \alpha_{i+1}\right) W_{i, i+1}^{m}\right. \\
& \left.+\sum_{i=1}^{N-2}\left(\alpha_{i} \alpha_{i+2}^{*}+\alpha_{i}^{*} \alpha_{i+2}\right) W_{i, i+2}^{m}+\sum_{i=1}^{N-3}\left(\alpha_{i} \alpha_{i+3}^{*}+\alpha_{i}^{*} \alpha_{i+3}\right) W_{i, i+3}^{m}\right\}
\end{aligned}
$$




\section{ACKNOWLEDGMENTS}

We are grateful to Robert Gordon for the programming. This work was supported in part by NSF Grant GK-42047.

\section{REFERENCES}

1. R. F. Harrington, "Field Computations by Moment Methods," Macmillan, New York, 1968.

2. J. R. Mautz and R. F. Harrington, Appl. Sci. Res. 20 (1969), 405-435.

3. R. MitrRA, Ed., "Computer Techniques for Electromagnetics," Pergamon Press, New York, 1973.

4. H. Well. and C.-M. ChU, Appl. Optics 15 (1976), 1832-1836.

5. D. E. Livesay, K. M. CheN, IEEE Trans. Microwave Theory Techn. MTT-22 (1974), 1273-1280.

6. P. Barber AND C. YeH, Appl. Optics 14 (1975), 2864-2872.

7. Edwin D. ReIlly, JR., J. Comput. Phys. 11 (1973), 463-492.

8. R. DeVore, D. B. Hodge, and R. G. Kouyoumuian, J. Appl. Phys. 42 (1971), 3075-3083. 